\title{
Involving Medical Students in Informed Consent: A Pilot Study
}

\author{
Sabaretnam Mayilvaganan ${ }^{1} \cdot$ Andrew G. Hill ${ }^{2}$
}

Published online: 19 October 2015

(C) Société Internationale de Chirurgie 2015

\section{Dear Sir}

We read with interest the article "Involving Medical Students in Informed Consent: A Pilot Study" by Chiapponi et al. [1]. We congratulate the authors on their study of an innovative approach for teaching 6th year Medical students the surgical consent process. However, we have two concerns that we would like the authors to address.

While the practice of residents obtaining consent from patients prior to surgery is no doubt common in many countries, this paper points out the danger in this practice. In many jurisdictions, the person doing the surgery is responsible for the surgical consent and while the signing of a form can be delegated, the consent process cannot. While the step intervention appears to be of use in teaching students how to obtain consent it is perhaps of more concern that the consent process by surgical residents, instead of experienced surgeons explaining surgery, was so ineffective. Whether this is because the patients feel apprehensive [2] when they discuss surgery or because of the resident's ability to fully inform the patient is not answered by this paper but is an avenue for further research.
Second, how exactly was consent obtained for students to participate in this study? It is stated that this was 'voluntary' but that can mean many things. Did medical students express any concern that they felt pressured to participate and did they truly understand that they were not the ones obtaining the consent from the patient? Given the poor understanding of the surgery that the student's discovered did the senior surgeon go and talk to the patient prior to surgery?

Thank you for your comments on these issues.

Sabaretnam Mayilvaganan

Andrew G. Hill

\section{References}

1. Costanza C et al (2015) Involving medical students in informed consent: a pilot study. World J Surg. doi:10.1007/s00268-0153090-9

2. Sherlock A, Brownie S (2014) Patients' recollection and understanding of informed consent: a literature review. ANZ J Surg 84(4):207-210
Sabaretnam Mayilvaganan drretnam@gmail.com

1 Department of Endocrine and Breast Surgery, Sanjay Gandhi Postgraduate Institute of Medical Sciences, Lucknow, India

2 Middlemore Hospital, Counties Manukau Health, University of Auckland, Auckland, New Zealand 